\title{
Immunotherapy in asthma
}

\author{
Jo A Douglass, Francis C K Thien, Robyn E O'Hehir
}

Department of Allergy and Clinical Immunology, Alfred Hospital, Prahran, Victoria, Australia

Introductory article

\section{Ragweed immunotherapy in adult asthma}

PS Creticos, CE Reed, PS Norman, J Khoury, NF Adkinson, CR Buncher, WW Busse, R Bush, J Gadde, JT Li, HB Richerson, RR Rosenthal, WR Solomon, P Steinberg, JW Yunginger

Background. Although allergen immunotherapy is effective for allergic rhinitis, its role in treating asthma is unclear. Methods. We examined the efficacy of immunotherapy for asthma exacerbated by seasonal ragweed exposure. During an observation phase, adults with asthma who were sensitive to ragweed kept daily diaries and recorded peak expiratory flow rates between July and October. Those who reported seasonal asthma symptoms and medication use as well as decreased peak expiratory flow were randomly assigned to receive placebo or ragweed-extract immunotherapy in doses that increased weekly for an additional two years. Results. During the observation phase, the mean (SE) peak expiratory flow rate measured in the morning during the three weeks representing the height of the pollination season was 454 (20) litres per minute in the immunotherapy group and 444 (16) litres per minute in the placebo group. Of the 77 patients who began the treatment phase, 64 completed one year of the study treatment and 53 completed two years. During the two treatment years, the mean peak expiratory flow rate was higher in the immunotherapy group (489 (16) litres per minute vs. 453 (17) in the placebo group $(p=0.06)$ during the first year, and 480 (12) litres per minute vs. 461 (13) in the placebo group ( $p=0.03$ ) during the second). Medication use was higher in the immunotherapy group than in the placebo group during observation and lower during the first treatment year $(p=$ $0.01)$ but did not differ in the two groups during the second year $(p=0.7)$. Asthma symptom scores were similar in the two groups $(p=0.08$ in year 1 and $p=0.3$ in year 2$)$. The immunotherapy group had reduced hayfever symptoms, skin test sensitivity to ragweed, and sensitivity to bronchial challenges and increased IgG antibodies to ragweed as compared with the placebo group; there was no longer a seasonal increase in $\mathrm{IgE}$ antibodies to ragweed allergen in the immunotherapy group after two years of treatment. Reduced medication costs were counterbalanced by the costs of immunotherapy. Conclusions. Although immunotherapy for adults with asthma exacerbated by seasonal ragweed exposure had positive effects on objective measures of asthma and allergy, the clinical effects were limited and many were not sustained for two years. (N Engl J Med 1996;334:501-6)

The results of the study by $\mathrm{C}$ reticos and colleagues ${ }^{1}$ in the introductory article have generated much controversy, with proponents as well as opponents of immunotherapy in asthma interpreting the results in their respective favour. This double blind, placebo controlled trial involved three centres near the Johns Hopkins University School of M edicine in M aryland and four centres near the Mayo Clinic in M innesota and was conducted over three years (including the first year conducted over three years (including the first year ervational year). Patients entering the treatment phase were selected for the presence of seasonal asthma symptoms during the ragweed season, and were randomised to receive placebo or immunotherapy during the subsequent two years.

The results showed an improvement in peak expiratory flow rates in the treatment group compared with the placebo group over the two years. Although there was a trend towards improved asthma symptoms in the treatment group during the first year, this was not sustained for the second year. Bronchodilator and inhaled corticosteroid usage was significantly lower in the treatment group during the first year of therapy, but the groups did not differ in this respect by the second year. In addition, there was evidence of the immunological efficacy of this treatment, with reduced skin test and bronchial challenge sensitivity to ragweed, increased and specific IgG antibodies to ragweed, and abolition of the
seasonal increase in IgE antibodies after two years of seasonal increase in IgE antibodies after two years of
treatment. Furthermore, hayfever symptoms were also reduced significantly over the two years.

The investigators were rigorous in their selection of patients and the allergen to evaluate the effect of immunotherapy in asthma. They chose to investigate a seasonal allergen, ragweed pollen, of which the major 
antigenic determinant A mb a I is well characterised, with standardised extracts available. Efficacy of immunotherapy with this allergen in the doses administered in this study had previously been demonstrated in ragweed sensitive seasonal rhinitis. ${ }^{2}$ In order to exclude confounding allergens, subjects were required to have a positive skin prick test for ragweed with less reactivity to other allergens. In addition, they had to have had asthma for more than one year, with seasonal symptoms and a positive reaction to methacholine challenge. H owever, as the study bears out, such patients are few, with only 127 of 1000 patients screened meeting these criteria. Only 90 subjects were eligible after an observational year (which included significant worsening of asthma symptom scores, worsening peak flow rates, and worsening medication scores). This figure was further reduced to 77 by dropouts before treatment was initiated. T his highly selected group was then randomised to receive active treatment or placebo with well matched demographic characteristics.

Significantly, the attrition rate in the subsequent two years was different in the placebo group (16 of 40 patients) from the immunotherapy group (eight of 37 patients). The attrition in the placebo group, secondary to a poor response, undoubtedly reduced the statistical power of the study, particularly in the second year. Hence the resul showing a beneficial effect are more likely to represen the true effects of immunotherapy. The apparent lack
of a sustained effect in the second year can be attributed of a sustained effect in the second year can be attributed to this high dropout rate in the placebo group, and is consistent with a regression towards the mean. Com patible with this, $\mathrm{C}$ reticos and colleagues, in reply to correspondence regarding the study, provide in formation that a group of 17 patients receiving immunotherapy for a third year continued to show a similar magnitude of improvement in symptoms, peak flows, and medication use. ${ }^{3}$

The use of patient diaries in recording medication usage and twice daily peak flow readings as outcome measures in clinical asthma studies has inherent pitfalls Inaccuracies with a tendency to inflate the peak flow rate and to record more medication use than actually occurred have been reported. While microprocessor based electronics for peak flow meters and inhalation devices have been developed, these were not available at the initiation of this study. N evertheless, within the limitations of the study and given the inherent variation of these outcome measures, the differences in peak flow readings and medication usage were significant compared with the control group. In addition, improvement was observed in objective parameters such as reduced skin test and bronchial challenge sensitivity to ragweed.

Adverse reactions occurred in seven of 37 patients receiving immunotherapy on 14 occasions. Five patients in the placebo group reported adverse reactions, but one of these was a treatment error due to the inadvertent administration of active extract. Only two subjects on active treatment dropped out after having several systemic reactions. The rates of allergic reactions in these asthmatic patients are similar to patients with rhinitis. While this finding may be interpreted as indicating that patients with asthma are at no greater risk of suffering adverse reactions than patients with rhinitis, the authors state in their discussion that asthmatic patients may still develop greater respiratory distress with a severe reaction. ${ }^{1}$ This study therefore does not shed any new light on the risk of adverse reactions during im- munotherapy in asthma, and supporters and detractors alike can find argument in their favour.

One of the features of this study was a cost benefit analysis of the respective treatments. While there was a reduced cost of medication in the immunotherapy group, this was counterbalanced by the cost of materials and injection charges for immunotherapy for the duration of the study. H owever, in this analysisit is unknown ation of the study. H owever, in this analysisit isunknown
whether there is any sustained benefit following the whether there is any sustained benefit following the
cessation of immunotherapy, which may translate into cessation of immunotherapy, which may
further cost savings in pharmacotherapy.

In summary, this paper does show immunological and clinical efficacy of specific immunotherapy in a highly selected group of seasonal asthmatic subjects with a predominant sensitivity to a seasonal allergen. $\mathrm{H}$ owever, the magnitude of the clinical effect is similar $\mathrm{H}$ owever, the magnitude of the clinical effect is similar
to that of various asthma medications such as inhaled to that of various asthma medications such as inhaled
steroids of moderate dosage or a long acting $\beta$ agonist. steroids of moderate dosage or a long acting $\beta$ agonist.
$M$ oreover, the savings in reduction of medication usage are counterbalanced by the cost of this treatment.

\section{Immunotherapy: current practice}

The use of immunotherapy for the management of allergic disease was pioneered by $\mathrm{N} \mathrm{oon}^{4}$ and $\mathrm{F}$ reeman ${ }^{5}$ in 1911 at St M ary's H ospital in L ondon. The efficacy of the therapy, practised for allergic rhinitis and conjunctivitis and maintained over a three year period, was not in doubt Since those orly times the clinical practice of immunoth of immunotherapy has evolved with more rigorous preparation and standardisation of allergen extracts, together with an increasing number of studies examining the efficacy of treatment to a wide range of allergens.

The efficacy of immunotherapy in seasonal allergic rhinitis is well established and generally considered beyond contention. ${ }^{6}$ A carefully controlled study conducted to examine the question of efficacy of immunotherapy in relation to grass pollen-induced allergic rhinitis revealed a threefold reduction in symptom scores and a fourfold reduction in medication use in patients undergoing active versus placebo immunotherapy with a biologically standardised grass pollen extract. ${ }^{7}$ This study has been used as the basis for the British Society of Allergy and Clinical Immunology guidelines which advocate immunotherapy as a treatment moda seasonal allergic rhinitis unresponsive to anti-allergic drugs.

Given the current evidence, immunotherapy is not recommended for the management of food allergy as it has not been proved to be effective and is contraindicated for safety reasons. Similarly, based on current evidence, administration of immunotherapy by routes other than subcutaneously is not recommended outher than in controlled research studies. Subcutaned immunotherapy is mandated for the treatment of lifethreatening allergic reactions to insect stings (bees and wasps). Other allergic conditions, particularly respiratory allergies, represent a relative indication for immunotherapy and are therefore the current subject of debate, research, and cost-efficacy analysis.

Several current practice guidelines in Europe and Australia emphasise the cardinal principles of immunotherapy: ${ }^{8}$

- The allergen is clearly defined in relation to the symptoms.

- The presence of IgE to that allergen is clearly documented by skin prick testing or quantitation of allergen-specific IgE.

- The extract available is potent, containing a measured 
amount of the major antigenic determinants of the allergen

- Mixtures of allergens are not endorsed for immunotherapy.

\section{Role of allergy in asthma symptoms}

The theoretical basis for the use of immunotherapy in asthma hinges on the significance of allergic sensitisation as a causative factor in continuing asthmatic symptoms. Epidemiological studies have clearly shown that sensitivity to some allergens is correlated with an increased incidence of asthma in children. This has been well documented, especially in relation to the house dust mite. ${ }^{10}$ Epidemiological associations also exist for exposure to cat, ${ }^{11}$ cockroach, ${ }^{12}$ and some mould antigens $s^{13}$ posure to cat, ${ }^{11}$ cockroach, ${ }^{12}$ and some mould antigens ${ }^{13}$
and the subsequent development of asthma early in life. Studies of environmental modification and their effect on asthma point to a role for ongoing exposure to allergens in the persistence of asthma in some individuals. ${ }^{14}$ Deaths from asthma have also been asStill further evidence for the role of allergy in asthma can be found in human models of allergen challenge whereby airflow obstruction is evoked by exposure to allergens. C onsequently, treatments for allergic disease have been directed to several sites in the allergic in flammatory response (fig 1 ).

The presence of asthma can therefore be clearly associated with allergic responses, but the relative role of allergens as causative agents in the persistence of asthma compared with other factors such as intrinsic bronchial hyperresponsiveness, genetic factors, and infections has yet to be determined and is likely to vary substantially between individuals. A clearer understanding of the relative contributions of allergy to asthma would lead to more accurate predictions of the potential efficacy of immunotherapy in individuals with asthma.

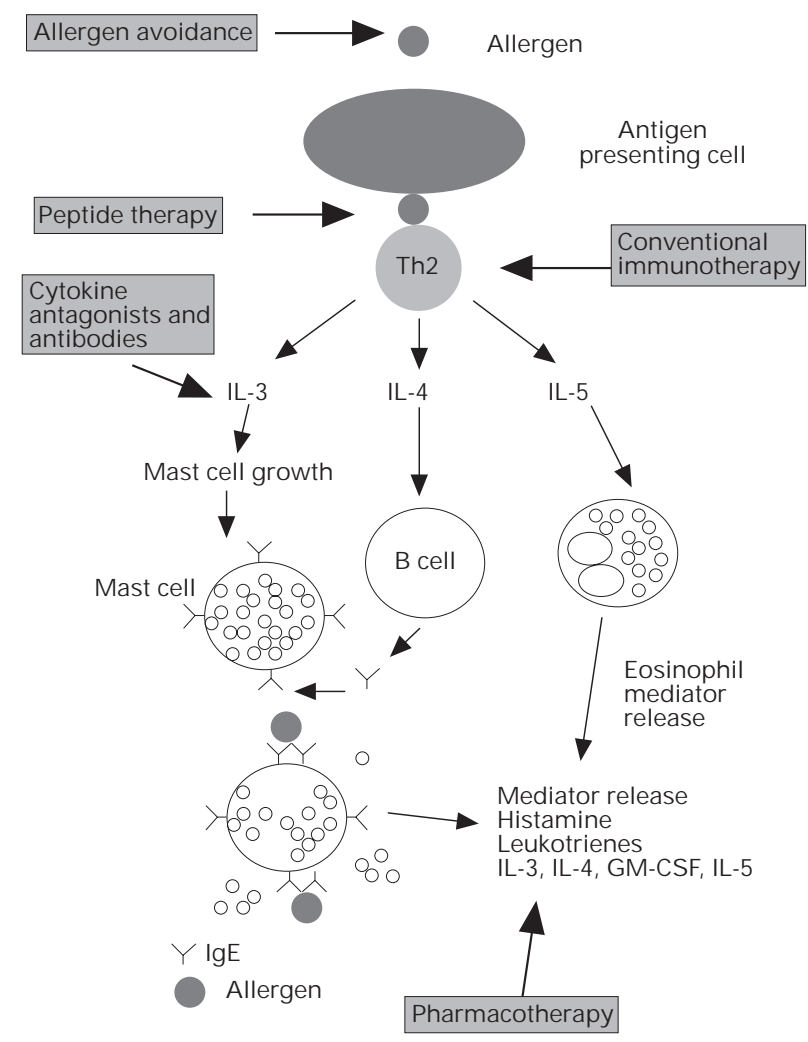

Figure 1 The allergic response may be modulated at

Figure 1 The allergic response may be
several points by therapeutic modalities.

\section{Efficacy of immunotherapy}

The efficacy of treatment for asthma can be judged on the basis of objective and subjective end points. One of the most reliable measurements is improvement in lung function measured at several time points during a trial by spirometry or in serial peak flow measurements observed over the time course of the study. These measurements, however, may be confounded by other measurements, however, may be confounded by other
simultaneous end points such as medication reduction. simultaneous end points such as medication reduction.
Furthermore, the reliability of peak flow measurement as a monitoring tool of patients' symptoms has been questioned. ${ }^{16}$

$M$ easurement of bronchial responsiveness to allergen or to non-specific stimuli such as histamine or methacholine provides some objective index of the sensitivity of the airways to exogenous stimuli. I nhalational allergen of theairways to exogenous stimuli. Inhalational allergen
challenge is a laboratory technique which intuitively bears some resemblance to the situation in "real life"; however, the degree of intrinsic airway responsiveness will determine the relationship of this measurement to symptoms. ${ }^{17} \mathrm{~F}$ urthermore, measurements of bronchial responsiveness do not correlate uniformly with asthma symptoms. ${ }^{18}$

$M$ ethods of evaluation may also be found in the sequential monitoring of symptoms and the assignation of a symptom and/or medication score for comparison between therapeutic modalities. Such an index may be confounded by the difficulties encountered by patients in accurately recording their symptoms, particularly for prolonged periods. In addition, the use of medications will modulate symptom severity.

Finally, in a fiscal age, the cost of treatment needs to be considered. H owever, treatment costs for the duration of a trial may not reflect the costs associated with treatments which have advantages over a period of time longer than the period of the trial. In this respect, the costs of immunotherapy are vulnerable to being overestimated in the relatively short time frames in which clinical trials are conducted.

\section{Clinical practice of immunotherapy for asthma:} the world view

International practice varies substantially in the clinical use of immunotherapy for asthma. Some guidelines acknowledge a clinical role for immunotherapy in asthma, particularly in younger patients and in those with a limited range of specific allergen sensitivities. ${ }^{19-21}$ Conversely, in the UK the use of allergen immunotherapy for asthma is not considered justified. ${ }^{22}$ What evidence is there that immunotherapy is efficacious in the management of asthma?

\section{Seasonal allergens}

TIST SENTITITY

Allergy to pollens is one of the most commonly encountered sensitivities in atopic individuals, being a major causative factor in seasonal allergic rhinoconjunctivitis. N evertheless, the epidemiological evidence that such allergy contributes significantly to asthma remains predominantly associative. R eid and coworkers ${ }^{23}$ found that hospital presentations with asthma closely correlated over a four year period with grass pollen counts. However, C reticos and colleagues ${ }^{1}$ screened the diary cards of approximately 1000 patients to identify 90 who had seasonal asthma with exacerbations temporally related to the ragweed pollen season. This might suggest that, for most patients, clear evidence of the contribution of pollen allergy to asthma 
symptoms is difficult to document, despite detection of allergen-specific IgE .

D espite these findings, several controlled trials of the efficacy of immunotherapy in asthma due to seasonal pollen antigens have been performed. M ost of the trials show an improvement in symptom scores following Immunological studies and bronchial provocation with Immunological studies and bronchial provocation with allergen were conducted in some of these studies and
most revealed a response to treatment with a reduction in bronchial responsiveness to allergen and increase in serum-specific lgG. In contrast, however, Bruce and co-workers reported a double blind, placebo controlled torical reports of allergic asthma in the ragweed season and corresponding skin test reactivity which showed no significant improvement in symptom scores for asthma, allergic rhinitis, nor a change in the provocative dose of allergen in inhalational challenge. ${ }^{26}$

T aken together, the evidence from the available trials to assess the efficacy of immunotherapy for pollen allergy in asthma points to an improvement in symptoms in several well conducted studies. L imited data also suggest an improvement in pulmonary function. Whether the an improvement in pulmonary function. Whether the extent of the clinical improvement detected is comparable to standard pharmacological therapies could be
answered by comparative trials and is considered in the introductory article by $\mathrm{C}$ reticos and colleagues, where the improvement in peak flow readings was found to be comparable to that with low dose inhaled corticosteroids.

\section{Perennial allergens}

HOUSE DUST MITES (DERMATOPHAGOIDES

PTERONYSSINUS AND D FARINAE)

The house dust mites ( $D$ pteronyssinus and $D$ farinae) have been recognised since the 1960s as the major perennial allergen sources relevant in allergic diseases ${ }^{27}$ E pidemiological studies have subsequently provided further compelling evidence of the association between ther compelling evidence of the association between An important study providing evidence for the critical role of exposure to house dust mite and the subsequent development of asthma in children is that by Sporik and co-workers who studied a group of children longitudinally, revealing that exposure of children to large amounts of house dust mite allergen is an important amounts of house dust mite allergen is an important determinant in the subsequent development of asthma. ${ }^{10}$ were performed in the 1960 s with poor clinical efficacy. ${ }^{2}$ In 1971 a study of immunotherapy with an extract of house dust mite compared with an extract of house dust reported a reduction in symptoms in those who received the hou the house dust mite extract but not in those who received the house dust extract. ${ }^{30}$ It was not until the most common house dust mites, $D$ pteronyssinus and $D$ farinae or, more importantly, their faecal particles were understood to contain the major antigenic components of house dust that trials of partially purified or modified (tyrosine or alum adsorbed) extracts were conducted.

Several controlled trials have shown the efficacy of immunotherapy for house dust mite allergic asthma determined by symptom reduction and medication use, $^{31-34}$ but other comparable trials have failed to reveal efficacy on these parameters. ${ }^{35-37}$ Several trials have also shown a reduction in bronchial challenge responsiveness to allergen. ${ }^{32} 36$

The most convincing index of efficacy in trials of immunotherapy in asthma remains pulmonary function,

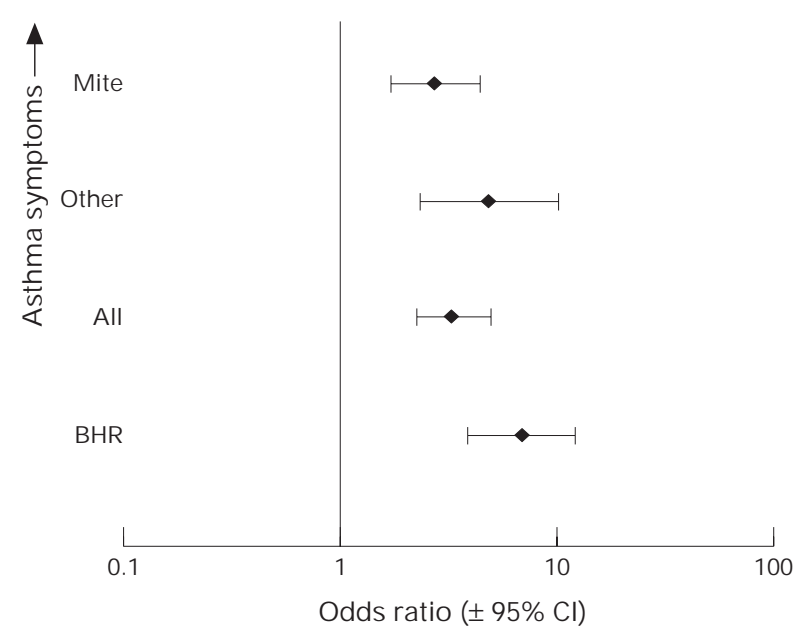

Figure 2 Odds ratio for improvement in asthma symptom or bronchial hyperresponsiveness (BHR) determined by a
meta-analysis of all double blind, placebo controlled trials of immunotherapy following house dust mite, other allergens combined (other), and all allergens (all). The odds ratio for improvement in bronchial hyperresponsiveness performed. Modified from all trials in which this was permission.

a parameter not universally observed as a major end point. A study by Bousquet et al ${ }^{38}$ revealed an improvement in peak flow, while a later controlled parallel group study by the same group, using an extract standardised for the antigen $\mathrm{D}$ er $\mathrm{p} \mathrm{I}$, revealed an improvement
in forced expiratory volume in one second $\left(F E V_{1}\right)$ of in forced expiratory volume in one second $\left(\mathrm{FEV}_{1}\right)$ of
$16 \%$ predicted in the actively treated group. ${ }^{39} \mathrm{~A}$ study by Price and co-workers also revealed an improvement in thoracic gas volume. ${ }^{33}$ These findings are in contrast with earlier studies using mite extracts which found no improvement in lung function 313236 or even a decline emerge in studies over time may be partly explained by the increasing recognition of the allergen content of extracts and thestandardisation of the extracts according to antigenic content.

The difficulty of determining the efficacy of immunotherapy in the context of simultaneous environmental control measures was highlighted by a recent double blind, placer ed study conducted by Peroni and colleagues on children in which the effects of immunotherapy could not be distinguished from those due to allergen avoidance obtained by both groups by use of treatment at high altitude. ${ }^{40}$

The efficacy of house dust mite immunotherapy in asthma was most thoroughly examined in the recent meta-analysis published by Abramson et a ${ }^{41}$ of double . This study found that house dust mite immunotherapy led to a statistically significant $4.5 \%$ improvement in lung function. M oreover, an additional 30 studies revealing a negative effect of immunotherapy for house dust mite would be needed to justify statistically the statement that it is of no benefit in asthma.

Given the major role of house dust mite antigens in the genesis of asthma several key questions remain unanswered by the trials published to date. Firstly, is immunotherapy more likely to be effective in children, preventing the subsequent onset of asthma by altering immunological responses to antigen? To date, one study utilising an unstandardised extract would suggest that this is so, but such an important question deserves further confirmation. ${ }^{42}$ Secondly, can a group of patients 
be identified who are more likely to benefit from immunotherapy? The study by Bousquet and colleagues ${ }^{39}$ suggests that young patients, those with mild to moderate asthma, and those with clear evidence of an extrinsic origin of their symptoms are more likely to benefit, and these conclusions were supported by the study by Warner et al. ${ }^{32}$ Thirdly, is there a likelihood study by Warner et al. ${ }^{32}$ Thirdly, is there a likelihood that extracts standardised for allergenicity could increase
the efficacy of treatment? Any studies designed to answer the efficacy of treatment? Any studies designed to answer
these questions must be large enough to provide suffithese questions must be large enough to provide suffi-
cient power for meaningful analysis. Pauli and colleagues have suggested that sample sizes of 40 per patient group in a blinded, placebo controlled study would be necessary to ensure adequate power. ${ }^{34}$

MOULDS

The role of moulds as an allergic trigger for asthma is suggested by epidemiological studies showing correlation of sensitivity to moulds with current asthma symptoms in children ${ }^{13}$ and the association between sensitivity and exposure to moulds and deaths from asthma ${ }^{43} \mathrm{Imm}$ unotherapy for mould sensitivity has been hampered by the lack of availability of standardised potent extracts, so few controlled trials have been performed. ${ }^{44} \mathrm{~N}$ evertheless, two controlled trials suggest an improvement in asthma symptoms and medication scores following immunotherapy with standardised mould extracts ${ }^{4546}$ A reduction in bronchial hyperresponsiveness to allergen was also observed 46 Taken together, these studies suggest that immunotherapy may be efficacious, but convincing evidence of improvement in pulmonary function is lacking. Further studies using standardised extracts may be warranted in those in dividuals with clear evidence of asthma secondary to mould exposure.

ANIMAL DANDERS

Several trials have been conducted to investigate the efficacy of immunotherapy in asthma induced by animal danders, particularly cat allergens. The efficacy of treatment appears to be best in trials using a monoclonal antibody standardised extract ${ }^{47}$ resulting in an improvement with immunotherapy of symptom scores and bronchial responsiveness to allergen. However, previ studies have not documented an improvement in symptoms in patients who have remained in close contact with cats. ${ }^{48} \mathrm{~N} 0$ efficacy has been noted with extracts to dogs in dog-sensitive patients. ${ }^{49} \mathrm{~T}$ he duration of efficacy of immunotherapy to animal danders was investigated in a five year follow up study of patients treated with cat or dog immunotherapy, and those individuals who underwent active desensitisation to cat extracts were found to have a durable decrease in asthma symptoms found to have a durable decrease in asthma symptoms ver that time period ${ }^{5}$

The spectrum of clinical sensitivity to $T$ cell epitopes of $\mathrm{Fel} \mathrm{d} \mathrm{I,} \mathrm{the} \mathrm{major} \mathrm{cat} \mathrm{allergen,} \mathrm{is} \mathrm{well} \mathrm{conserved}$ throughout the population allergic to cats. In view of this, cat allergen was used for the first trial of a peptide vaccine aimed at the amelioration of symptoms of cat allergy. The clinical trials of $F$ l $d$ I peptide immunotherapy have reported conflicting results with varying efficacy at different centres. ${ }^{5152}$ Adverse reactions to the vaccine of late onset were particularly puzzling and concerning. H owever, large protein determinants were administered rather than epitope based vaccines which could perhaps result in increased immunogenicity. F uture developments of this form of immunotherapy using smaller peptides may be more effective.

\section{Combination allergen immunotherapy}

Although not recommended as state of the art, mixtures of allergens are frequently used as immunotherapy, particularly in the USA. Indeed, the introductory article by Creticos and colleagues on the efficacy of ragweed immunotherapy in asthma concluded that combinations of allergens may be more efficacious than single antions of allergens may be more efficacious than single antigen therapy. ${ }^{1}$ Adkinson et al ${ }^{21}$ have recently published the results of a large double blind, placebo controlled study of 120 children with asthma randomised to receive either placebo or immunotherapy with up to seven relevant antigens for a period of 24 months. End points were the amount of medication required for asthma methacholine responsiveness, peak methacholine responsiveness, peak flow readings, a reduction in medication use in both placebo and active treatment groups to a similar degree; the only advantage to the active group was the use of fewer inhaled corticosteroids and a slight improvement in peak flow readings which just reached statistical significance. These results therefore do not justify the use of combinations of allergen immunotherapy in children. It does suggest that ap-

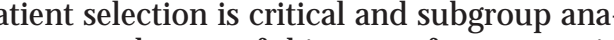
lysis would support the use of this type of treatment in younger patients and those with less severe asthma. This concurs with other publications. ${ }^{39} \mathrm{M}$ ixtures of allergens are therefore not currently recommended as best practice for immunotherapy in Europe or Australia.

\section{Safety of immunotherapy for asthma}

Local reactions to immunotherapy are common, occurring in up to $25 \%$ of patients, and do not constitute a contraindication to therapy. $L$ arge local reactions should prompt an alteration of the dosage schedule.

Concerns regarding the safety of immunotherapy are particularly pertinent to the treatment of asthma given the information that most deaths from immunotherapy have occurred in individuals suffering from asthma. The have occured in individuals suffering from asthma. The 1986 report of the British Committee of Safety of M edicines, ${ }^{53}$ which reported that 29 individuals, 16 of whom were undergoing immunotherapy for asthma, had died due to severe allergic reactions secondary to immunotherapy over 29 years, highlighted issues of the safety of immunotherapy and prompted its virtual withdrawal from common medical practice in the UK. Other reports from the USA, where immunotherapy is administered by allergy specialists, indicated greater safety. ${ }^{54}$ It was also evident from the $C$ ommittee's report that modification of the vaccines, such as by alum precipitation, greatly improved the safety of immunotherapy.

In the study by $\mathrm{C}$ reticos et $\mathrm{al}^{1}$ seven patients receiving ragweed therapy developed systemic reactions that required treatment while four in the placebo group were also treated for side effects; thus, approximately $10 \%$ of those on active therapy suffered a systemic reaction at some stage. The recent study reported by Adkinson and co-workers utilising desensitisation to multiple allergens in children ${ }^{21}$ revealed a rate of 2.6 systemic reactions per 100 injections, with all children responding to treatment for the adverse reaction. Comparably, data gathered from specialist physicians in the UK report an incidence of one systemic adverse reaction per 500 injections. ${ }^{6}$ T he U K data found that significant systemic reactions exclusively occurred within 45 minutes of administration of the extract.

These data would support the overall safety of immunotherapy in the treatment of asthma provided that it is administered to patients with stable asthma and 


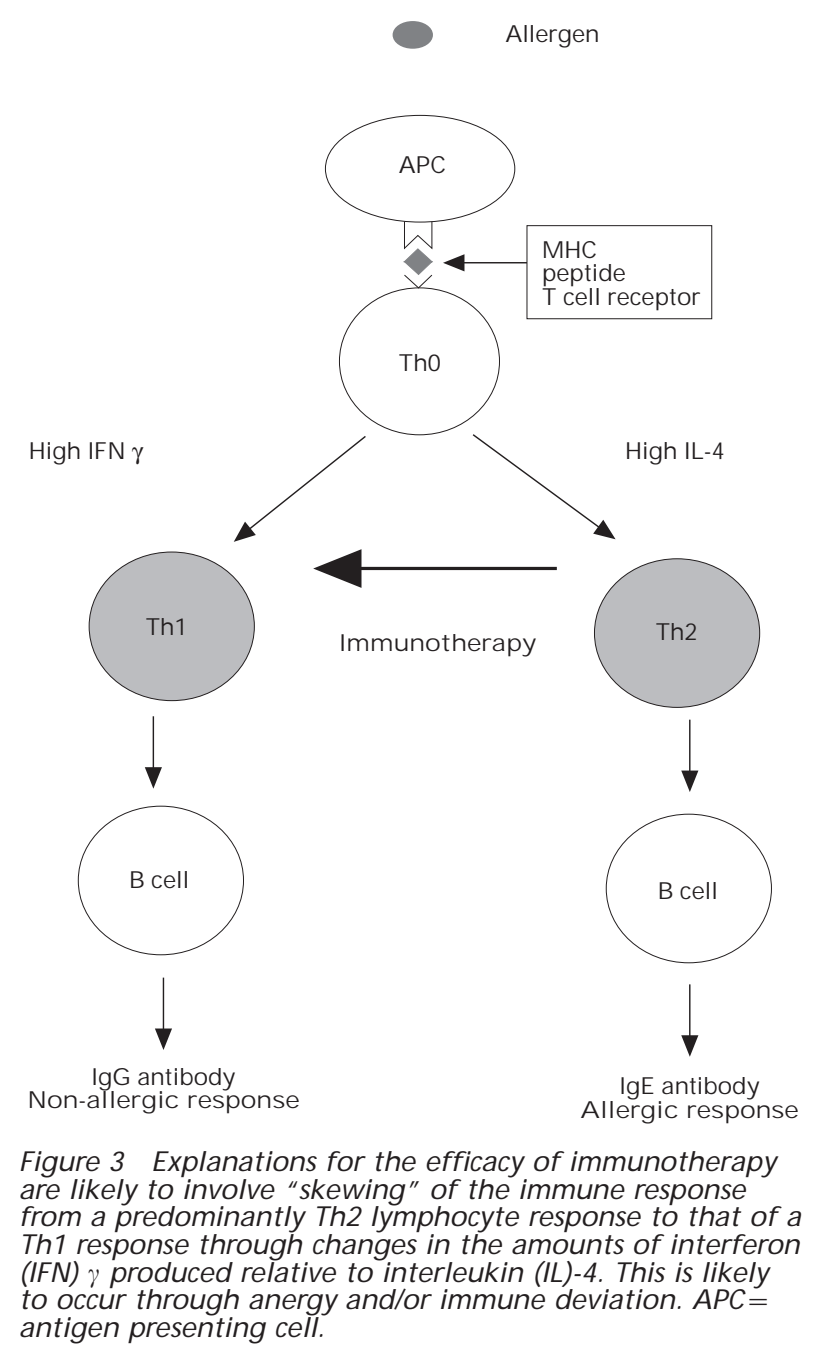

that they are instructed to wait in the doctor's surgery for 45 minutes following allergen administration.

Bousquet and co-workers have shown that modulation of the schedule of administration of immunotherapy together with co-administration of antihistamine and glucocorticoid premedication can substantially reduce the incidence of adverse reactions. ${ }^{55}$

\section{Immunology}

Immunological studies investigating the mechanism of effective immunotherapy following the parenteral administration of allergen in the human model are limited A number of different hypotheses have been proposed to explain the observed effectiveness of allergen immunotherapy including increased membrane stability of ody levels, increased competitive IgG antibodies, the generation of suppressor T cells, and the induction of anti-idiotypic networks (fig 3). Since allergen-specific IgE antibodies are a characteristic feature of the allergic immune re sponse, early studies investigating the mechanisms of immunotherapy focused on changes in allergen-specific antibodies. Levels of immunoglobulin $\mathrm{E}$ antibody usually decrease, but generally not until some month or years after treatment and with little relationship to clinical response. In fact, many studies show an initial increase in levels of specific IgE. M ost studies investigating $T$ cell reactivity following allergen immunotherapy report decreased allergen-specific proliferation. ${ }^{56-58}$ This change would be consistent with the induction of antigen-specific non-responsiveness (anergy) and/or the generation of new subsets of allergen-specific $T$ cells with altered reactivity to the allergen. Several studies have reported a decrease in allergeninduced production of interleukin (IL )-4 following immunotherapy but variable results have been obtained with interferon gamma (IF N $\gamma$ ) and other cytokines 5859 Although these studies suggest a net switch in $\mathrm{T}$ cell cytokine production from Th2 to Th1 type, the underlying mechanism has not been investigated. Possibilities include the induction of antigen-specific non-responsiveness (anergy) with selective manipulation of the cytokine profile, or changes in the cytokine milieu driving the differentiation of naive $\mathrm{T}$ cells along a Th1 pathway (immune deviation) (fig 3). M urine allergy pathay (immune devition) (fig 3 ). Murine allergy to the Th1 pathway can redirect differentiation towards tolerance (IgG) rather than hypersensitivity (IgE) reactions. ${ }^{60} \mathrm{~F}$ urthermore, some clinical studies have found raised serum-specific levels of IgG, IgA, and altered cytokine profiles of $\mathrm{T}$ cells infiltrating the skin after challenge in successful allergen-mediated de sensitisation ${ }^{61}$ It is likely that clonal anergy, immune deviation or, indeed, may be responsible for the observed clinical efficacy of allergen immunotherapy.

\section{Recommendations}

The first modality of treatment for asthma should be pharmacotherapy to achieve and stabilise best lung function. The identification of trigger factors and their avoidance comprise a major secondary goal of treatment and are particularly important in those with clear allergic precipitants for their asthma. I mmunotherapy should
be seen as a possible therapeutic adjunct in well assessed be seen as a possiblet
and selected cases.

The introductory article by $\mathrm{C}$ reticos and colleagues demonstrates immunological and clinical efficacy in a carefully selected group of patients with seasonal allergies over a 12 month period. N evertheless, the magnitude of clinical effect is similar to that of inhaled nitude of clinical effect is similar to that of inhaled
steroids in moderate doses or of a long acting $\beta$ agonist. steroids in moderate doses or of a long acting $\beta$ agonist.
A comparison of the relative costs of therapy depends on the time frame in which it is considered. The benefits in the short term (up to three years) are not substantially greater than those that might be achieved with optimal use of pharmacotherapy, which is without the potential of anaphylactic reactions. However, if benefit is sustained for 5-10 years then the savings compared with pharmacotherapy are substantial.

An additional factor in favour of immunotherapy in children is its potential to modify the immune response as a form of primary preventative therapy. Limited evidence suggests that subsequent development of asthma can be prevented by childhood grass pollen immunotherapy. Although this is an attractiveargument, immunotherapy. Although thisis an attractive argument, convincing data from large cohort studies have yet to
be published. This would argue against the routine clinical use of immunotherapy for young children with asthma. Active research and clinical trials are currently exploring the use of a vaccine to decrease the risk of subsequent allergen sensitisation. C areful selection of appropriate infants based on family history, infantile food allergy, and possible genetic screens as predictive atopic loci become identified is essential (P Holt, personal communication).

The studies presented here indicate the need for further clinical research of specific immunotherapy in broader groups of allergic asthmatic subjects of well characterised phenotype to identify specifically those 
LEARNING POINTS

* Respiratory allergic diseases constitute a relative rather than an absolute indication for immunotherapy.

* Immunotherapy to house dust mite, pollens, cat dander, and some moulds have been show $\mathrm{n}$ to be effective in the management of upper and low er respiratory tract symptoms.

* Immunotherapy should be considered as an adjunct to pharmacotherapy in well assessed and selected cases of asthma.

* Treatment with immunotherapy should only be used in selected patients where symptoms are attributable to a single predominant inhaled allergen and there is evidence of $\operatorname{lgE}$ production by the patient to the suspected allergen.

* The reported incidence of systemic reactions to immunotherapy varies from approximately 1:30 to 1:500 injections, almost invariably occurring within 45 minutes of administration of the allergen extract.

* Immunotherapy should only be administered where resuscitation equipment and medication are available to treat systemic reactions immediately.

individuals most likely to respond favourably to this treatment. Until such large clinical trials have been carried out, the benefits, risks and costs of immunotherapy compared with pharmacotherapy for asthma cannot be established.

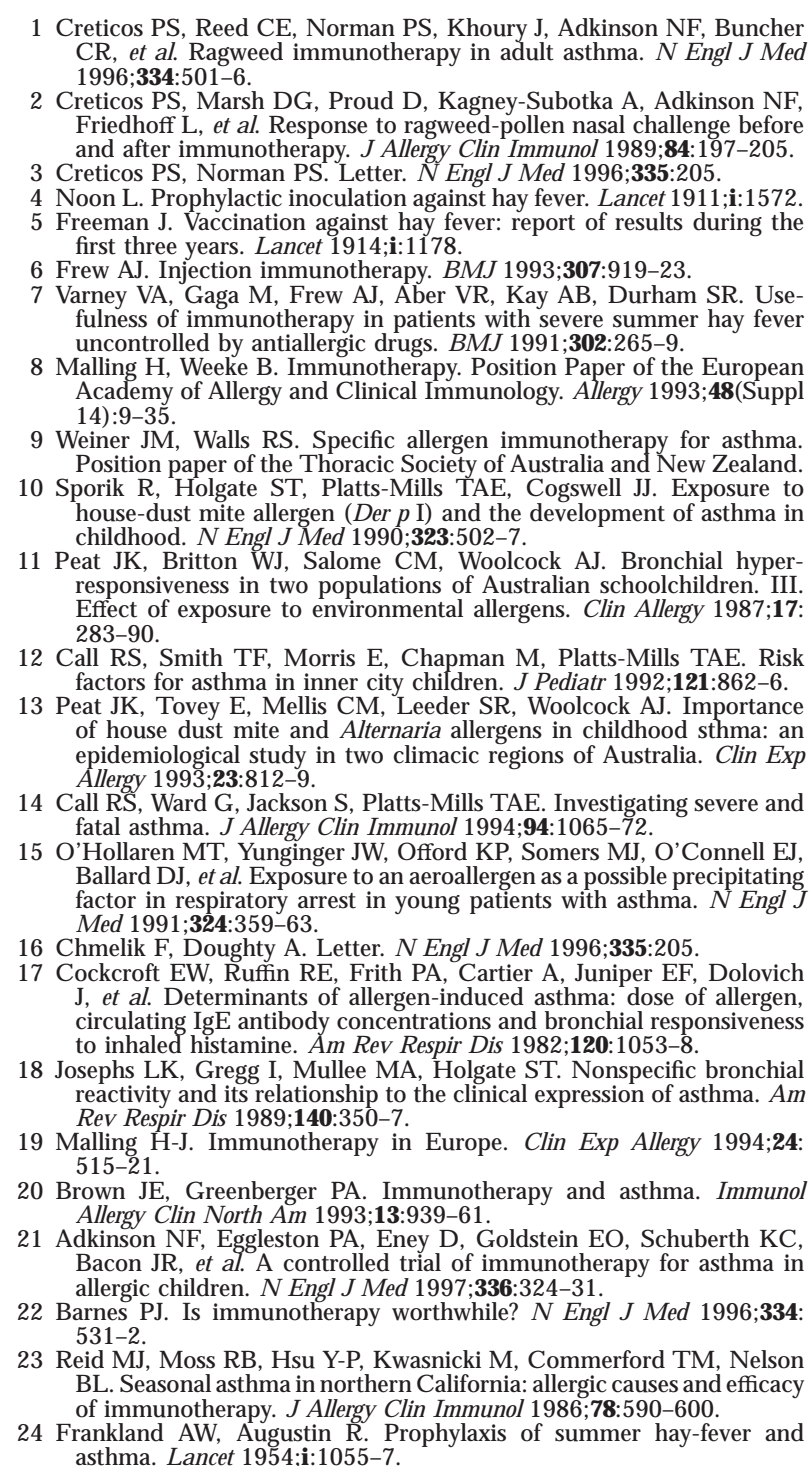

25 Ortolani C, Pastorello E, M oss RB, H su Y-P, Restuccia M, Joppolo G, et al. G rass pollen immunotherapy: a singleyear double-blind, placebocontrolled study in patients with grass pollen-induced asthma and
rhinitis. A Allergy Clin I mmunol 1984; $3: 283-90$. rhinitis. A Allergy Clin Immunol 1984; 73:283-90.
26 Bruce A, Norman PS, Rosenthal RR, Lichtenstein LM. The role of
rogweed pollen in autumnal asthma, J Allergy Clin Immunol 1977:59. ragweed pollen in autumnal asthma. J Allergy Clin Immunol 1977;59.
449-59. 27 M aunsell K M. M ites and house dust allergy in bronchial asthma. Lancet

28 Turner KJ, Stewart GA, Woolcock AJ, Green W, Alpers M P. Re lationship between mite densities and the prevalence of asthma: Resarch Committee of the British Tuberculosis A ssociation. Treatment of house dust allergy. BM $1968 ; 3: 774-7$.

M aunsell $K$, Wraith $D G, H$ ughes $A M$. H yposensitisation in mite asthma. Lancet $1971 ;$ i: $: 967-8$.
Smith AP. Hyposensitization with Dermatophagoides pteronyssinus an-

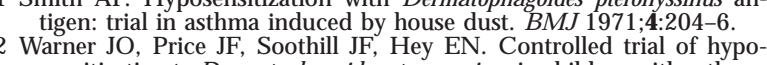
sensitisation to Dermatophagoides pteronyssinus in children with asthma. Lancet 1978;ii: : 12-5.
. H ey EN. Turner M W, Soothill JF. A controlled

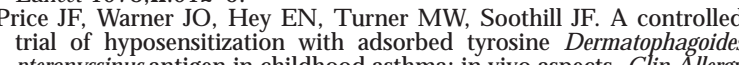
pteronyssinus antigen in childhood astha.
1984; 14:209-19.

4 Pauli G, Bessot JC, Bigot H, Delaume G, Hordle DA, Hirth C, et al. Clinical and immunologic evaluation of tyrosine adsorbed Dermatrial. J A Allergy Clin Immunol 1984; 74:524-35.
Gaddie J, Skinner C C P Palmer K N V. Hyposensitisation with house dust
mite vain

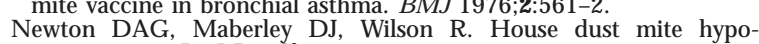
sensitization. B I J D is Chest 1978;72:21. $M$ ite Allergy Subcommittee of the Research committee of the British
Thoracic Association. A trial of house dust mite extract in bronchial ousquet J, Calvayrac P G uerin B, Hejiaoui A, D hivert H, H ewitt B, et al. Immunotherapy with a standardised Dermatophagoides pteronyssinus extract. A H rop Cin Brociek W, et al. Specific immunotherapy with a standardized D erma tophagoides pteronyssinus extract. . A llergy Clin 1 mmunol 1988;82:971-7. Ment

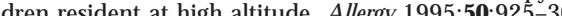
41 Abramson M J, Puy RM, Weiner JM. Is allergen innunotherapy effective in asthma? A m J Respir Cri

Johnstone DE, Dutton A. The value of hyposensitization therapy for
bronchial asthma in children: a 14 year study. Pediatrics $1968: 42$

73 Call RS, Ward G, Jackson S, Platts-M ills TAE. Investigating severe and
fatal Salvaggio JE. Burge HA Chapman IA Emerging concepts in mold immunotherapy: what is the role of immunotherapy? J Allergy Clin Immunol 1993;92:217-2.
H orst M, H ejjaui A, H orst V, M ichel FB, Bousquet J. D ouble-blind, placebo-controlled rush immunoth extract. J Allergy Clin I mmunol 46 D reborg S, Agrell B, Foucard T, Kjellman N IM, K oivikko A, Nilsson a purified and standardised Cladosporium herbarum preparation. Allergy 1986;41:131-40.
Alvarez-C esta E, Cuesta-H erranz J, Puyana-Ruiz J, Cuesta-H arranz
C, Blanco-Q uiros A. M onoclonal antibody-standardised cat extract immunotherapy: risk-benefit effects from a double-blind placebo study. I Allergy Clin Immunol 1994; 93:556-66.

48 Bousquet J. Specific immunotherapy in asthma: is it effective? I Allergy 
49 Valovirta $E$, K oivikko $A$, Vanto $T$, Viander $M$, Ingemen $L$. Immunotherapy in allergy to dog: a double blind clinical study. An
A llergy 1984;53:85-8. 50 H edlin G, H eilborn $H$, Lilja G, Norrlind K, Pegelow $K O$, Schou C,
al. Long-term follow-up of patients treated with a three year course al. Long-term follow-up of patients treated with a three year 89-85. $\mathrm{N}$ orman $P S$, O Ohman $J L$, Long AA, Creticos PS, G efter M A, Shaked
$Z$, et al. Treatment of cat allergy with T-cell reactive peptides. Am J Respir Crit C are M ed 1996; 154:1623-8

peptides: effect on skin tests and cytokine synthesis in cat-allergic human subjects. Int I mmunol 1996;8:1937-45.

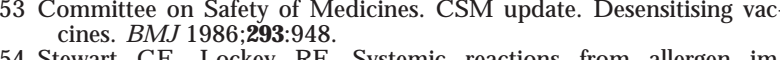
cines. BM J 1986;293:948. Systemic reactions from
munotherapy. I A Allergy Clin Immunol 1992;90.567-78.

$5 \mathrm{H}$ ejaoui $A$, Dhivert $H$, M ichel FB, Bousquet $\mathrm{J}$. I mmunotherapy with a standardized Dermatophagoides pteronyssinus extract. IV. Systemic
reactions according to the immunotherapy schedule. I Allergy Clin Immunol 1990;85:473-9.
56 Gatien JG, M erler E, Colten HR. Allergy to ragweed antigen E: effect
of specific immunotherapy on the reactivity of human T lymphocytes in vitro. Clin 1 unotherapy on the reactivity of human Tymphocytes 57 A koum $\mathrm{H}$, Tsicopoulos, Vorng $\mathrm{H}$. Venom immonotherapy modulates

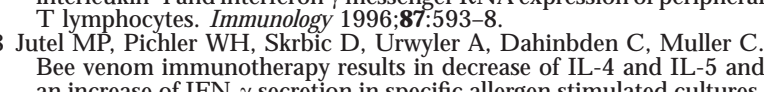

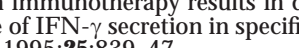
59 Secrist $H$, D D R ruyff $H$, U metsu $D$. Interleukin-4 production by CD 4 + cells from allergic individuals is modulated by antigen concentraton $H$ sieh $C S$, $H$ eimberger $A B$, G old $J S, O{ }^{\prime} G$ arra A, M urphy KM. Differ4 and 10 in $a n$ of Thelper phenotype development by interleukin

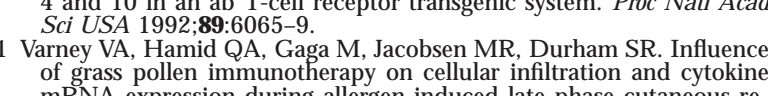
mRNA expression during allergen-induced
sponses. I Clin Invest 1993;92:664-51. 